\title{
Un análisis de las relaciones entre funciones ejecutivas, lenguaje y habilidades
}

\section{matemáticas}

\section{An analysis of the relationship between executive functions, language and math skills}

\author{
Alicia Risso, Manuel García, Montserrat Durán, Juan Carlos Brenlla, Manuel Peralbo y Alfonso Barca \\ Universidad de A Coruña
}

\begin{abstract}
Resumen
El objetivo de este trabajo fue explorar la relación existente entre un conjunto de variables cognitivolingüísticas (conciencia fonológica, competencia lectora y matemática) y las funciones ejecutivas (FE), en las etapas iniciales de escolarización. Con la finalidad de hacer un estudio exploratorio se seleccionaron 16 niños y niñas de 7 y 8 años, cursando $2^{\circ}$ de Educación Primaria en un colegio público. Los datos sobre conciencia fonológica y competencia lectora se obtuvieron con el LOLEVA y el Índice de Competencia Matemática con el TEMA-3; los relacionados con las FE se obtuvieron con el CHEXI pasado al profesorado. Los resultados muestran que tanto las medidas cognitivas como las lingüísticas tienen una alta correlación con las funciones ejecutivas evaluadas, pero no en todas las tareas ni con el mismo peso. También entre las medidas de los dominios lingüístico y matemático parecen existir claras relaciones, posiblemente mediadas por su dependencia básica de las funciones ejecutivas.
\end{abstract}

Palabras Clave: Funciones ejecutivas, conciencia fonológica, competencia lectora, habilidades matemáticas.

\begin{abstract}
The aim of this study was to explore the relationship between a set of cognitive-linguistic variables (phonological awareness, reading and math skills) and executive functions (EF), in the initial stages of schooling. In order to make an exploratory study 16 children aged 7 and 8 years old studying 2nd of Primary Education in a public school were selected. Data on phonological awareness and reading skills were obtained with the LOLEVA; Math's Competence Index was assessed with TEMA-3 and those relating to the FE were obtained with Chexi (teachers' version). The results show that both cognitive and linguistic measures are highly correlated with executive functions, although there are differences depending on tasks, and the magnitude of correlations are not homogeneus. Moreover, there were significant relationships between measures of linguistic and mathematical domains, which possibly are mediated by their dependence on executive functions.
\end{abstract}

Keywords: executive functioning, phonological awareness, reading skills, math skills
Entre los procesos o funciones de carácter no lingüístico implicados en el aprendizaje lector y en la adquisición de las destrezas matemáticas básicas destacan, sin duda, los procesos ejecutivos. Éstos incluyen, siempre y desde cualquier aproximación teórica, algunos de tanta importancia como la memoria de trabajo, el control inhibitorio y la flexibilidad cognitiva. Funciones, todas ellas, situadas en el córtex prefrontal, aunque existen otras zonas también implicadas en el funcionamiento ejecutivo como, por ejemplo, los ganglios basales (inhibición), el cerebelo (control motor) o el cuerpo calloso (comunicación interhemisférica prefrontal).

Las Funciones Ejecutivas (FE) abarcan el conjunto de procesos que subyacen a la conducta consciente $\mathrm{y}$ planificada dirigida a metas, a las respuestas en las situaciones novedosas o difíciles y a la capacidad de inhibir aquellas conductas que nos alejan del objetivo perseguido, por lo que se refiere a lo necesario para el control deliberado del pensamiento, de las emociones y de las acciones. Como señala Carlson (2005), el funcionamiento ejecutivo se refiere a procesos cognitivos de alto nivel, autorregulatorios, que ayudan en la supervisión y control del pensamiento y la acción. Estas habilidades incluyen: control inhibitorio, planificación, flexibilidad atencional, corrección y detección de errores y resistencia a la interferencia.

No hay un acuerdo total sobre cómo definir el funcionamiento ejecutivo, aunque el modelo de mayor acuerdo quizá sea el de Miyake, Friedman, Emerson, Witki y Howerter (2000). Para ellos existen tres aspectos básicos en el funcionamiento ejecutivo: inhibición de las respuestas dominantes, actualización y supervisión de las representaciones en la memoria de trabajo, y cambio entre tareas o conjuntos mentales.

Será precisamente en el marco establecido por Miyake, donde situaremos esta investigación, si bien no conviene olvidar que Hughes, Ensor, Wilson y Grahan (2010), en un estudio longitudinal con niños entre 4 y 6 encontraron que sus resultados se pueden explicar desde un factor único.

\section{FE y conciencia fonológica}

Los procesos metalingüísticos se encuentran, también, bajo el control del lóbulo prefrontal. La conciencia fonológica, como es sabido, se refiere a la habilidad para operar explícitamente sobre los segmentos fonológicos del lenguaje. Es el conocimiento consciente de que las 
palabras están compuestas de varias unidades de sonido (Gillam y van Kleecl, 1996), así como la capacidad para reflexionar y manipular sobre las subunidades del lenguaje hablado: sílabas, unidades intrasilábicas y fonemas (Treiman, 1991). En un sentido amplio, abarcaría las habilidades para manipular las palabras que componen las frases (conciencia lexical), las sílabas de las palabras (conciencia silábica), y las unidades más pequeñas de la palabra, los fonemas (conciencia fonémica) (Defior, 1996). No existe unanimidad entre los investigadores al definir cuál es la unidad lingüística que puede ser incluida en el término conciencia fonológica. Algunos utilizan el término para referirse estrictamente al fonema, mientras que otros incluyen además la sílaba. Para Treiman (1991), por su parte, es la conciencia de cualquier unidad fonológica, ya sean sílabas, unidades intrasilábicas o fonemas, $\mathrm{y}$, según esto, propone un modelo de jerarquías de conciencia fonológica con niveles que van desde conciencia silábica, hasta fonémica pasando por intrasilábica.

Lo que parece claro, en todo caso, es que la mediación fonológica es un proceso fundamental en los primeros años del aprendizaje de la lectura. El nivel de conciencia fonológica que alcance un niño durante el inicio del aprendizaje es esencial para el nivel lector que alcanzará durante los años siguientes. La conciencia fonológica es, por tanto, un factor crítico para el aprendizaje de la lectura, pues se debe tener conocimiento sobre emparejar unidades sonoras y gráficas, ya que será necesario que los niños desarrollen la capacidad para analizar la estructura fonológica de la lengua para tener un buen desarrollo lector (Alegría, 2006).

Las habilidades fonológicas se inician a partir de los 3 o 4 años, por lo que a esta edad los niños ya estarían capacitados para analizar los segmentos fonológicos del habla, es decir, segmentar en varias unidades de sonido (Márquez y de la Osa, 2003). Considerando la influencia de las habilidades lectoras a largo plazo en el desarrollo lector, se podría decir que la conciencia fonológica indica la capacidad lectora y está en la base de las diferencias entre buenos y malos lectores. Existen varias vías para el desarrollo del conocimiento fonológico, una podría ser aprender a leer y escribir en un sistema de escritura alfabético y otra, la enseñanza explícita de habilidades fonológicas (Hulme, Snowling, Caravolas, y Carroll, 2005), por lo que el conocimiento fonológico y el aprendizaje de la lectura estarían relacionados de forma bidireccional.

De forma muy resumida, las conclusiones más importantes que se extraen sobre el desarrollo de la conciencia fonológica son las siguientes: a) Existe una progresión evolutiva en el desarrollo de las habilidades fonológicas, iniciándose a los 4-5 años, de modo que primero aparece la capacidad para manipular las palabras, seguida de la de manipular sílabas y fonemas; b) Existe un efecto de la posición que ocupa la unidad a manipular en la palabra, de modo que es más difícil la manipulación de las unidades en posición final que inicial; (c) El tipo de estructura silábica $(\mathrm{CV}, \mathrm{VC}, \mathrm{CCV}$, etc.) puede afectar a la complejidad de la tarea; d) A través del entrenamiento a edades tempranas, las habilidades fonológicas pueden mejorar; e) El éxito del aprendizaje inicial de la lectura depende de las habilidades fonológicas; y f) Existe una relación bidireccional entre conciencia fonológica y lectura.

Dado que el aprendizaje en la escuela tiene lugar a través de la lectura, aquellos que aprenden antes pueden tener un mejor rendimiento escolar. Los problemas en el aprendizaje lecto-escritor afectan al posterior aprendizaje de los contenidos escolares y por ello al rendimiento en las materias del currículo escolar (Bryant \& Bradley, 1985). Podemos decir entonces, que el aprendizaje de la lectura y escritura exige un esfuerzo metalingüístico, ya que hace necesario que los niños accedan primero al código fonológico para poder utilizar las unidades sonoras y gráficas.

\section{FE y dificultades de aprendizaje lector}

Swanson, Zheng y Jerman (2009), en su meta-análisis sobre memoria a corto plazo, memoria operativa y dificultades de lectura, concluyen que en la base de las dificultades de lectura se puede encontrar un modelo de déficit verbal en el que se fracasa al utilizar eficientemente los recursos tanto del sistema ejecutivo como fonológico. Esta idea de que las FE pueden estar relacionadas con las dificultades de lectura, pero también con las actividades de segmentación, lleva a pensar que ambas estarán altamente intercorrelacionadas. También en otros trabajos (como el de Conners, 2009) se encuentra una relación importante entre control atencional y comprensión lectora, lo que permite pensar que el control inhibitorio se suma a las habilidades de decodificación y de comprensión para explicar el rendimiento en comprensión lectora. Además, como ya se indicó anteriormente, la conciencia fonológica, por su carácter metalingüístico, tiene un papel predictivo crucial en el desarrollo de la lectura. Farrar y Ashwell (2008) estudiaron su relación con otras habilidades lingüísticas y cognitivas, en particular la flexibilidad representacional. De hecho analizaron el papel de la teoría de la mente, el desarrollo del lenguaje y el funcionamiento ejecutivo en la emergencia de la conciencia fonológica en niños preescolares antes del desarrollo de la lectura y confirmaron la existencia de claros vínculos, como el existente entre la comprensión de la teoría de la mente y la habilidad de rimar. En un sentido similar se dirigían los trabajos de Bental y Tirosh (2007), entre otros, que confirman el importante papel que las funciones controladas por el córtex prefrontal tienen en el desarrollo metalingüístico y, por ello, en el desarrollo de la conciencia fonológica. Lo importante, en todo caso, es que un fracaso en la utilización tanto del sistema ejecutivo como del fonológico puede producir dificultades de lectura. La disfunción ejecutiva puede estar relacionada con problemas de comprensión lectora en su desarrollo inicial (Peralbo, Brenlla, García, Barca y Mayor, 2012), puesto que constituyen requisitos importantes para resolver problemas de forma eficaz.

\section{FE y desarrollo de habilidades matemáticas}

Como señalan Cardoso y Cerecedo (2008) una competencia matemática se vincula con el ser capaz de hacer, relacionado con el cuándo, cómo y por qué utilizar determinado conocimiento como una herramienta. Las 
dimensiones que abarca el ser matemáticamente competente son: a) Comprensión conceptual de las nociones, propiedades y relaciones matemáticas; b) Desarrollo de destrezas procedimentales; c) Pensamiento estratégico: formular, representar y resolver problemas; d) Habilidades de comunicación y argumentación matemática, y e) Actitudes positivas hacia las situaciones matemáticas y a sus propias capacidades matemáticas (Chamorro, 2003). Para Nunes y Bryant (2005) solamente aquella persona que reconozca las reglas lógicas puede entender y realizar adecuadamente incluso las tareas matemáticas más elementales.

De las diversas nociones y habilidades matemáticas que los niños deben adquirir a lo largo de la educación infantil y los primeros años de primaria, se encuentran en lugar destacado las relacionadas con la capacidad de seriar (seriación simple, múltiple e inferencia transitiva), clasificar (clasificación simple, múltiple e inclusión de clases) y comprender el concepto de número (principio cardinal). Esta última se encuentra en la base de la aparición de las operaciones de medida. Numerosas investigaciones han indagado la relación entre FE en Educación Infantil y el desempeño académico en Matemáticas (Bull y Scerif, 2001; Espy et al, 2004; Bull, Espy y Wiebe, 2008). Mencionaremos tres de ellas por su interés para la presente investigación.

Bull, Espy y Wiebe (2008) hallaron que el desempeño en tareas de memoria a corto plazo y FE (control inhibitorio, flexibilidad cognitiva y planificación) se hallaba asociado a un mejor rendimiento inicial de los niños en las habilidades matemáticas y de lectura. Asimismo, dicha superioridad en el rendimiento académico se sostenía a lo largo de los años de la Educación Primaria. Por otro lado, el rendimiento en tareas de memoria viso-espacial, durante la etapa de Educación Infantil, sería un buen predictor de su capacidad matemática durante la Educación Primaria posterior. En particular, la memoria viso-espacial y la memoria de trabajo operaban como predictores del desempeño matemático infantil en todos los períodos en los cuales éstos fueron evaluados. El resto de las FE (control inhibitorio, flexibilidad, planificación) actuaban como indicadores de la capacidad de aprendizaje en general. Es decir, no se hallarían asociados al rendimiento de un dominio específico.

Por su parte, Geary, Hoard, Byrd-Craven, Nugent y Numtee (2007) realizaron un estudio en el cual evaluaron la relación entre el rendimiento de niños en pruebas matemáticas y su desempeño en tareas de memoria de trabajo y velocidad de procesamiento. Encontraron que los niños con un rendimiento normal en matemáticas eran más rápidos y tenían mayor precisión a la hora de responder a tareas que presentaban demandas de identificación de conjuntos numéricos, recuperación y retención de información numérica, estimación lineal y capacidad de conteo. Asimismo, la capacidad de reconocimiento de conjuntos numéricos se hallaba relacionada con el desempeño en tareas de memoria de trabajo viso-espacial, y los niños con dificultades procesaban dicha información de modo más lento, requiriendo de un esfuerzo mayor en la determinación del tamaño de los conjuntos.
Desde otra perspectiva, Blair y Razza (2007) llevaron a cabo una investigación longitudinal en la cual estudiaron la relación existente entre algunas FE (control de la atención, control inhibitorio) y el rendimiento en tareas de Matemática y Lengua. Los niños fueron evaluados dos veces: la primera vez entre los 3 años nueve meses y los 5 años 8 meses, y la segunda entre los 5 años 7 meses y los 6 años 11 meses. Específicamente, se evaluaron las capacidades de control (factor temperamental asociado al autocontrol), comprensión de creencias falsas, control inhibitorio, cambio de atención e inteligencia. Los resultados de la primera medición no predecían el desempeño de los niños en las tareas de reconocimiento fonológico y de letras. No obstante, las puntuaciones en control inhibitorio se hallaban vinculadas al desempeño en las tareas matemáticas durante ese periodo. En la segunda medición las puntaciones en control inhibitorio, cambio de atención e inteligencia, se hallaban asociadas a la capacidad lectora de los niños (reconocimiento fonético y de conocimiento de letras). Asimismo, las puntuaciones en control inhibitorio, cambio de atención, inteligencia y comprensión de falsas creencias durante esta etapa estaban vinculadas a la capacidad matemática.

Tales resultados indicarían que la influencia del Funcionamiento Ejecutivo sobre el desempeño académico dependería tanto de la competencia académica implicada como del período evolutivo considerado. Por tanto, se trata de considerar, como lo más importante, que el niño realice una manipulación de los objetos matemáticos, desarrolle su creatividad, reflexione sobre su propio proceso de pensamiento a fin de mejorarlo, adquiera confianza en sí mismo, se divierta con su propia actividad mental, haga transferencias a otros problemas de la ciencia y de su vida cotidiana y por último, prepararlo para los nuevos retos de la tecnología (Guzmán, 2007).

Dada la importancia que las FE parecen tener como soporte inicial de otros procesos cognitivo-lingüísticos, el objetivo de este trabajo fue analizar las relaciones entre estos procesos en una muestra de niños de $2^{\circ}$ curso de Educación Primaria (EP), donde se suponen desarrollados y automatizados estos procesos.

\section{Método}

\section{Participantes}

La muestra estaba compuesta por 16 niños y niñas de $2^{\circ}$ de EP de un colegio público, con edades comprendidas entre los 7 y 8 años. En todos los casos se obtuvo el consentimiento informado de sus padres.

\section{Variables}

1. Relacionadas con la conciencia fonológica evaluada con las correspondientes subescalas del LolEva:

Lectura de letras mayúsculas: aciertos

Lectura de letras minúsculas: aciertos

Lectura de palabras regulares: aciertos

Lectura de palabras complejas: aciertos

Lectura de pseudopalabras: aciertos

Segmentación de palabras en frases: aciertos y tiempo

Aciertos totales en la subescala de conciencia fonológica 
Aciertos totales en las tareas de conciencia silábica Aciertos totales en las tareas de conciencia fonémica

2. Relacionadas con las FE medidas a través del CHEXI:

Memoria de Trabajo (mínimo 13 máximo 65): Ítems $n^{\circ}: 1,3,6,7,9,12,14,17,19,20,21,23,24$

Control Inhibitorio (mínimo 11 máximo 55): Ítems $\mathrm{n}^{\mathrm{o}}: 2,4,5,8,10,11,13,15,16,18,22$

3. Índice de Competencia Matemática (ICM), proporcionado por el test TEMA-3

\section{Instrumentos}

La recogida de datos se realizó a través de los siguientes instrumentos:

1. LolEva (Peralbo et al., 2015): Es una prueba informatizada para la evaluación de la conciencia fonológica y la competencia lectora inicial en niños de preescolar y primeros niveles de primaria. Consta de dos subescalas, ambas con buena fiabilidad $(\alpha=.94$ para la de conciencia fonológica, y $\alpha=.92$ para la de competencia lectora inicial). La subescala de conciencia fonológica incluye tareas de rima y adición, omisión e identificación de sílaba y fonema, en posiciones inicial y final.

2. TEMA-3 (Ginsburg, Baroody, Núñez y Lozano, 2007). Mide la competencia matemática en niños de 3 a 8 años, así como en alumnos de más edad con problemas para aprender las matemáticas. Evalúa conceptos y habilidades formales e informales en diferentes campos: conteo, comparación de números, lectura de números y signos, dominio de hechos numéricos, habilidades de cálculo y comprensión de conceptos. Proporciona, a partir de estas pruebas, una puntuación categorial de Competencia Matemática (ICM-Cat), y un Índice de Competencia Matemática (ICM).

3. CHEXI (Thorell y Nyberg, 2008). Se trata de un cuestionario para padres y maestros que ha resultado de gran utilidad para la identificación precoz de síntomas relacionados con el TDAH (Thorell, Eninger, Brocki, e Bohlin, 2010; Thorell, Veleiro, Siuc y Mohammadid, 2013). Fue validado con dos muestras de 130 y 62 niños y niñas de entre 4 y 7 años, procedentes de poblaciones escolares de dos localidades suecas. La fiabilidad testretest fue adecuada $(r=.89)$, así como la consistencia interna, mientras que la correlación padres-maestros fue moderada (.32 para el factor Memoria de Trabajo y .38 para el factor Control Inhibitorio). Las correlaciones entre los factores del CHEXI y las pruebas cognitivas empleadas también fueron moderadas: .26-.39 entre el factor Memoria de Trabajo y una prueba de memoria de dígitos, y .28-.35 entre el factor de Control Inhibitorio y una tarea go/no-go.

\section{Procedimiento}

La aplicación de las pruebas se realizó en una sala preparada para ello, en la que se disponía de un ordenador portátil con el LolEva instalado, en el que se podían escuchar con claridad las instrucciones del evaluador/a. A continuación se aplicaba TEMA-3 en formato lápiz y papel. El CHEXI era cubierto por el profesor/a del niño/a. El tiempo medio de aplicación oscilaba entre 60 y 75 minutos.

\section{Resultados}

\section{Habilidades matemáticas y FE}

Como se puede ver en la Tabla 1, las dos FE evaluadas correlacionan de forma significativa con el índice de competencia matemática que proporciona el TEMA-3. Así, a mayores dificultades de memoria de trabajo y de control inhibitorio, peores resultados en las habilidades matemáticas evaluadas por el test, siendo la correlación más alta en el caso de la memoria de trabajo.

\section{Conciencia fonológica y $\mathrm{FE}$}

En el caso de la conciencia fonológica, la contribución de ambas FE es significativa en el caso del total de aciertos en la subescala de conciencia fonológica. Por otro lado, el total de conciencia fonémica correlaciona de forma significativa con la memoria de trabajo, y no con control inhibitorio.

\section{Lectura y FE}

En el caso de la competencia lectora, los resultados muestran una correlación alta con las dos FE en el caso de la lectura de letras mayúsculas y la lectura de palabras irregulares, mostrando que a mayores dificultades en estas funciones, peor es el resultado en lectura. En cambio, los análisis realizados no muestran correlaciones significativas con los tiempos de lectura. Además, considerados los totales de conciencia fonológica, por un lado, y de las tareas de lectura, por otro, se puede decir que una buena competencia lingüística se relaciona con un buen funcionamiento ejecutivo

Tabla 1

Correlaciones entre las variables cognitivo-lingüisticas $y$ las funciones ejecutivas $(N=16)$

\begin{tabular}{lcc}
\hline & $\begin{array}{c}\text { Memoria } \\
\text { de } \\
\text { Trabajo }\end{array}$ & $\begin{array}{c}\text { Control } \\
\text { Inhibitorio }\end{array}$ \\
\hline Mayúsculas & $-.511^{*}$ & $-.511^{*}$ \\
Minúsculas & -.359 & -.332 \\
Palabras regulares & $\mathrm{ET}$ & $\mathrm{ET}$ \\
Palabras complejas & $-.511^{*}$ & $-.511^{*}$ \\
Pseudopalabras & .170 & .170 \\
Separación de palabras & -.177 & -.392 \\
Total conciencia silábica & $-.660^{* *}$ & $-.521^{*}$ \\
Total conciencia fonémica & $-.635^{* *}$ & -.392 \\
Total conciencia fonológica & $-.627^{* *}$ & $-.502^{*}$ \\
Total tareas de lectura & -.233 & -.388 \\
Índice de competencia matemática & $-.689^{* *}$ & $-.601^{*}$ \\
\hline
\end{tabular}

$* p<.05, * * p<.01 ; \mathrm{ET}=$ efecto techo

\section{Intercorrelaciones entre las variables cognitivo- lingüísticas}

Los análisis de correlación realizados muestran, como era de suponer, una relación estadísticamente significativa entre la competencia matemática y los procesos lingüísticos evaluados. Como se puede ver en la Tabla 2, existen relaciones tanto en las dos subescalas, como en los totales proporcionados por el LolEva. 
Tabla 2

Intercorrelaciones entre conciencia fonológica, competencia lectora inicial y habilidades matemáticas

\begin{tabular}{lc}
\hline & $\begin{array}{c}\text { Índice de competencia } \\
\text { matemática }\end{array}$ \\
\hline Mayúsculas & .393 \\
Minúsculas & .328 \\
Palabras regulares & $\mathrm{ET}$ \\
Palabras complejas & .393 \\
Pseudopalabras & -.084 \\
Separación de palabras & .453 \\
Total conciencia silábica & $.747^{* *}$ \\
Total conciencia fonémica & $.656^{* *}$ \\
Total conciencia fonológica & $.729^{* *}$ \\
Total aciertos tareas de lectura & $.499^{*}$
\end{tabular}

$* p<.05, * * p<.01 ; \mathrm{ET}=$ efecto techo

\section{Discusión y conclusiones}

Los resultados muestran una relación directa $y$ significativa entre el nivel de funcionamiento ejecutivo y el resultado alcanzado en conciencia fonológica y en competencia matemática. Un buen funcionamiento ejecutivo parece asociarse a una buena capacidad para identificar los segmentos del habla y a un uso eficiente de ese conocimiento. Estos resultados son coherentes con los encontrados por Bental y Tirosh (2007), que confirman el importante papel que las funciones controladas por el córtex prefrontal tienen en el desarrollo metalingüístico y por ello en el desarrollo de la conciencia fonológica. También van en la línea de los resultaos obtenidos por Mayor, Fernández, Tuñas, Zubiauz y Durán, M. (2012), en una muestra de $1^{\circ}$ de Enseñanza Primaria. Sus datos mostraban la existencia de una correlación y capacidad de predicción significativa entre ambos tipos de variables.

De igual modo, la memoria de trabajo y el control inhibitorio parecen imprescindibles para el desarrollo de las habilidades matemáticas relacionadas con el conteo, la comparación y lectura de números y los signos, el dominio de los hechos numéricos, habilidades de cálculo y la comprensión de conceptos. Los resultados del presente trabajo indican que la relación entre FE y habilidades matemáticas se mantiene a lo largo de la escolaridad inicial. No sólo las FE son importantes para la adquisición de conceptos y operaciones matemáticas como ya señalaban Bull, Espy y Wiebe (2008), Geary et al. (2007) o Blair y Razza (2007), sino también para su mantenimiento y mejora.

Los procesos lectores se ven influidos por las FE cuando éstas están afectadas. En este nivel educativo, donde la lectura está ya automatizada, los procesos lectores que muestran una mayor afectación por la disfunción ejecutiva son los implicados en la identificación de letras (mayúsculas en este caso) y los procesos de conversión grafema-fonema característicos de la ruta fonológica. Es decir, aquellos niños con un bajo funcionamiento ejecutivo manifiestan dificultades lectoras en dos de sus procesos más básicos. Estos resultados coinciden con los de Peralbo, Brenlla, García,
Barca y Mayor, 2012) con una muestra de 63 estudiantes de $1^{\circ}$ de EP. Sus resultados revelaban la existencia de correlación y capacidad de predicción significativa entre el nivel de funcionamiento ejecutivo y la competencia lectora del alumnado, siendo mayor la influencia del primero cuanta más complejidad revisten los procesos lectores.

Por último, las correlaciones existentes entre conciencia fonológica y competencia matemática, con la quizás sorprendente ausencia de relaciones significativas con la competencia lectora, parecen indicar que es el elemento reflexivo, más metacognitivo, que forma parte misma de la conciencia fonológica, el determinante de otros aprendizajes posteriores. Esto parece deducirse del hecho de que entre conciencia fonológica y lectura exista una correlación significativa, y del mismo efecto entre conciencia fonológica y competencia matemática.

Globalmente los resultados se podrían interpretar en el sentido de que las FE están en la base de aprendizajes posteriores, como la conciencia fonológica, cuya correcta evolución contribuye a un buen aprendizaje en dos dominios independientes y específicos, como son el de la lectura y el de la matemática. Dicho de otro modo, el proceso evolutivo seguiría una dirección que iría del desarrollo de los procesos de dominio general, al de los procesos específicos de dominio cuyo correcto desarrollo se asienta sobre el nivel alcanzado en los ateriores.

Las limitaciones derivadas del tamaño de la muestra hacen necesario un estudio de mayor amplitud que aumente la potencia estadística de los análisis realizados.

\section{Referencias}

Alegría, J. (2006). Por un enfoque psicolingüístico del aprendizaje de la lectura y sus dificultades-20 años después. Infancia y Aprendizaje, 29, 93-111. http:// dx.doi.org/10.1174/021037006775380957

Bental, B., y Tirosh, E. (2007). The relationship between attention, executive functions and reading domain abilities in attention deficit hyperactivity disorder and Reading disorder: A comparative study. Journal of Child Psychology and Psychiatry, 48, 455-463. http://dx.doi.org/10.1111/j.1469-7610.2006.01710.x

Blair, C., \& Razza, R.P. (2007). Relating effortful control, executive function, and false belief understanding to emerging math and literacy ability in kindergarden. Child Development, 78, 647-663. http://dx.doi.org/10.1111/j.1467-8624.2007.01019.x

Bryant, P., \& Bradley, L. (1985). Children's reading problems. (Trad. Cast.: Problemas infantiles de lectura. Madrid, Alianza, 1998).

Bull, R., \& Scerif, G. (2001). Executive function as a predictor of children's mathematics ability: Inhibition, switching, and working memory. Developmental Neuropsychology, 19, 273-293. http://dx.doi.org/ 10.1207/S15326942DN1903_3

Bull, R., Espy, K.A., \& Wiebe, S.A. (2008). Short-term memory, working memory, and executive functioning in preschoolers: longitudinal predictors of mathematical achievement at age 7 years. Developmental Neuropsychology, 33, 205-228. http://dx.doi.org/10.1080/87565640801982312 
Cardoso, E.O. y Cerecedo, M.T. (2008). El desarrollo de las competencias matemáticas en la primar infancia. Revista Iberoamericana de Educación, 47, 1-11. http:// www.rieoei.org/deloslectores/2652Espinosav2.pdf

Carlson, S.M. (2005). Developmentally sensitive measures of executive function in preschool children, Developmental Neuropsychology, 28, 595-616. http://dx.doi.org/10.1207/s15326942dn2802_3

Chamorro, M. (2003). La didáctica de la matemática para primaria. Madrid: Síntesis Educación.

Conners, F.A. (2009). Attentional control and the simple view of reading. Reading and Writing, 22,591-613. http://dx.doi.org/10.1007/s11145-008-9126-x

Defior, S. (1996). Una clasificación de las tareas utilizadas en la evaluación de las habilidades fonológicas y algunas ideas para su mejora. Infancia y Aprendizaje, 19, 49-63. http://dx.doi.org/10.1174/ 02103709660560546

Espy, K.A., McDiarmid, M.M., Cwik, M.F., Stalets, M.M., Hamby, A. y Senn T.E. (2004). The contribution of executive functions to emergent mathematic skills in preschool children. Developmental Neuropsychology, 26, 465-86. http://dx.doi.org/10.1207/s15326942dn 26016

Farrar, M. J., \& Ashwell, S. (2008). The role of representational ability in the development of phonological awareness in preschool children. En K. B. Cartwright (Ed.), Literacy Processes: Cognitive Flexibility in Learning and Teaching (pp. 71-89). New York, NY: Guilford Press.

Geary, D. C., Hoard, M. K., Byrd-Craven, J., Nugent, L., \& Numtee, C. (2007). Cognitive mechanisms underlying achievement deficits in children with mathematical learning disability. Child Development, 78, 1343-1359. http://dx.doi.org/10.1111/j.1467-8624. 2007.01069.x

Gillam, R. \& van Kleeck, A.(1996). Phonological awareness training and short-term working memory: Clinical implications. Topics in Language Disorders, 17, 72-81. http://dx.doi.org/10.1097/00011363-1996 11000-00008

Herbert Ginsburg, H.; Baroody, A.J.; Núñez, M.C., \& Lozano, I. (2007). TEMA-3: Test de Competencia Matemática Básica. Madrid: TEA.

Guzmán, M. de (2007). Enseñanza de las Ciencias y la Matemática. Revista Iberoamericana de Educación, 43, 19-58. http://www.rieoei.org/rie43a02.pdf

Hughes, C., Ensor, R., Wilson, A., \& Graham, A. (2010). Tracking executive function across the transition to school: A latent variable approach. Developmental Neuropsychology, 35, 20-36. http://dx.doi.org/10. 1080/87565640903325691

Hulme, C.; Snowling, M.; Caravolas, M.; \& Carroll, J. (2005) Phonological skills are (probably) one cause of success in learning to read: A comment Castles and Coltheart. Scientific Studies of Reading, 9, 351-365. http://dx.doi.org/10.1207/s1532799xssr0904_2

Márquez, J. y de la Osa, P. (2003). Evaluación de la conciencia fonológica en el inicio lector. Anuario de
Psicología, 34, 357-370. http://www.raco.cat/index. php/AnuarioPsicologia/article/viewFile/61745/88530

Mayor, M.A., Fernández, M.L., Tuñas, A. Zubiauz, B., \& Durán, M. (2012). La relación entre funciones ejecutivas y conciencia fonológica en educación primaria. En L. Mata, F. Peixoto, J. Morgado, J. Castro, \& V. Monteiro (Eds). Educaçao, aprendizajem $e$ desenvolvimento: olhares contemporáneos a través da investigaçao e da práctica (pp. 1792-1806). Lisboa: ISPA.

Miyake, A., Friedman, N. P., Emerson, M. J., Witzki, A. H., \& Howerter, A. (2000). The unity and diversity of executive functions and their contributions to complex "frontal lobe" tasks: A latent variable analysis. Cognitive Psychology, 41, 49-100. http://dx.doi.org/ 49-100. 10.1006/cogp.1999.0734

Nunes, T., \& Bryant, P. (2005). Las matemáticas y su aplicación: La perspectiva del niño. México: Siglo XXI.

Peralbo, M., Brenlla, J.C., García, M., Barca, A. y Mayor, M.A. (2012). Las funciones ejecutivas y su valor predictivo sobre el aprendizaje inicial de la lectura en educación primaria. En L. Mata, F. Peixoto, J. Morgado, J. Castro y V. Monteriro (Eds.). Educaçao, aprendizajem e desenvolvimiento: olhares contemporáneos a través da investigaçao e da práctica (pp.76-90). Lisboa: IPSA.

Peralbo, M., Mayor, M.A., Zubiauz, B., Risso, A., Fernández, M.L. y Tuñas, A. (2015). The Loleva Oral and Written Language Test: Psychometric Properties. Spanish Journal of Psychology, 18, E18. http:// dx.doi.org/10.1017/sjp.2015.15

Swanson, H. L., Zheng, X., y Jerman, O. (2009). Working memory, short-term memory, and reading disabilities: A selective meta-analysis of the literature. Journal of Learning Disabilities, 42, 260-287. http://dx.doi.org/10.1177/0022219409331958

Thorell L.B., Eninger L., Brocki K.C., Bohlin G. Childhood Executive Function Inventory (CHEXI): A promising measure for identifying young children with ADHD? Journal of Clinical and Experimental Neuropsychology, 32, 38-43. http://dx.doi.org/10. 1080/13803390902806527

Thorell, L.B., \& Nyberg L. (2008). The childhood executive functioning inventory (CHEXI): a new rating instrument for parents and teachers. Developmental Neuropsychology, 33, 536-52. http:// dx.doi.org/10.1080/87565640802101516

Thorell, L.B., Veleiro, A., Siuc, A.F.Y., \& Mohammadid, H. (2013). Examining the relation between ratings of executive functioning and academic achievement: Findings from a cross-cultural study. Child Neuropsychology, 19, 630-638. http://dx.doi.org/10. 1080/09297049.2012.727792

Treiman, R. (1991). Phonological awareness and its roles in learning toread and spell. En: D. J. Sawyer \& B. J. Fox (Eds.), Phonological awareness in reading. The evolution of current perspective (pp. 159-189). New York: Spinger-Verlag. 